\title{
Las prácticas cotidianas frente a los dispositivos de la movilidad. Aproximación política a la movilidad cotidiana de las poblaciones pobres periurbanas de Santiago de Chile
}

Yves Jouffe. Doctor en Sociología del ENPC, Francia. Investigador, postdoctorado en el Instituto de la Vivienda. Universidad de Chile, Santiago.

Alejandra Lazo Corvalán. Magíster en Antropología, EHESS, Francia. Doctorado (c) Universidad de Toulouse - Pontificia Universidad Católica de Chile.

RESUMEN | Luego de su reforma, el sistema de transporte de Santiago de Chile aparece como inadecuado a las necesidades de los habitantes de los territorios social y espacialmente marginados. Los pobladores hacen uso de sus márgenes de maniobra para compensar esta inadecuación. Sus prácticas de movilidad cotidiana y sus maneras de utilizar, subvertir o de resistir al sistema de transporte serán confrontadas a los dispositivos que las modelan. Para calificar las formas de movilidad cotidiana en un barrio pobre de la periferia meridional de Santiago de Chile, serán distinguidos tres tipos de prácticas entendidas como relaciones de poder: liberación, libertad y libración.

PALABRAS ClAVE | Movilidad, periferia urbana, pobreza, transporte urbano.

ABSTRACT | In spite of the reforms in the transport system of Santiago de Chile, it appears that the needs of social and spatially marginalised inhabitants are not being met adequately. These poor city dwellers use their options for manoeuvre to offset this inadecuacy in their daily mobility. Their daily mobility practices and their ways of using, subverting or resisting the transport system are critically discussed in terms of the apparatus that shape the system. Three types of practices as power relations are identified - liberation, liberty and libration - and used to characterize forms of daily mobility in a poor district on the southern periphery of Santiago de Chile.

KEY WORDS | Mobility, urban periphery, poverty, urban transportation.

Recibido el 29 de agosto de 2009 , aprobado el 6 de enero de 2010.

Este artículo fue realizado en el marco del proyecto Fondecyt n ${ }^{\circ} 3080068$ sobre la vivienda como pilar de la movilidad cotidiana y la emancipación. Las entrevistas realizadas corresponden a parte del terreno de investigación doctoral de Alejandra Lazo sobre la movilidad cotidiana y el anclaje en Santiago de Chile.

Correspondencia: Yves Jouffe, Portugal 84 Santiago, Chile. Fono (56-2) 9783037. E-mail: yves.jouffe@gmail.com | Alejandra Lazo Corvalán, 5 allées Antonio Machado. 31058 Toulouse. Cedex 9. Laboratoire CIEU. Maison de la Recherche. Fono: 05.61.50.42.50.E-mail: alel55@yahoo.es 


\section{Introducción}

La nueva escala espacial alcanzada por la ciudad de Santiago de Chile (Hidalgo, 2007) ha generado dependencias funcionales entre zonas residenciales y lugares de trabajo. Este hecho a transformado y fragmentado los territorios de la movilidad cotidiana (Le Breton, 2004). En particular, se han impuesto viajes muy largos a una parte importante de la población. Sin embargo, los individuos disponen de un margen de maniobra que les permite adaptar su forma de movilizarse haciendo frente a esta nueva forma de ciudad. Estas adaptaciones parecen convertirse en verdaderas necesidades sobre todo para las comunas más pobres y periféricas de la urbe, como es el caso estudiado en la comuna de La Pintana.

En el año 2007 se implantó un nuevo sistema de transporte en la ciudad de Santiago, el llamado Transantiago, el cual afectó significativamente a estas poblaciones. En efecto, el sistema anterior de "micros amarillas" si bien presentaba un cierto desorden y escasa regulación, éste cubría la mayor parte del territorio de la ciudad, operaba con una elevada amplitud horaria, prestaba un servicio con un elevado nivel de frecuencia y facilitaba el acceso de la población a los distintos lugares en un solo trayecto.

Luego de la puesta en marcha de Transantiago y en la actualidad, los individuos han debido re-adaptar sus prácticas cotidianas en relación a la movilidad cotidiana que trata de establecer este nuevo sistema sin conformarse necesariamente con lo previsto por los planificadores del transporte. Este margen de organización de la movilidad cotidiana podría ser interpretado como una resistencia al sistema de transporte urbano y éste considerado como un dispositivo de control de la población. Nos interesa para objeto de este artículo analizar las prácticas de movilidad de los individuos frente a los dispositivos de movilidad impuestos.

Nos parece pertinente para este análisis retomar el concepto de dispositivo planteado por Michel Foucault interrogando las relaciones de poder. Comenzaremos nuestra discusión con una breve descripción del contexto del transporte en la ciudad de Santiago para luego desarrollar, en una segunda parte, el concepto de dispositivo y su nexo con la problemática de la movilidad cotidiana y el caso de Santiago. En un tercer apartado, construiremos tres categorías de análisis de las prácticas como relaciones de poder, las que llamaremos: liberación, libertad y libración. Finalmente, aplicaremos estas categorías a nuestro caso de estudio en la comuna de La Pintana al poner en evidencia los márgenes de maniobra que revelan las prácticas de movilidad cotidiana frente al sistema de transporte existente.

\section{La promesa del Transantiago y las prácticas de movilidad cotidiana}

La liberación del transporte público en Santiago de Chile producida en la década de los años ochenta, estuvo marcada por una flota gigantesca de microbuses pintados de amarillo que cubrían el paisaje de la ciudad. Este sistema de pequeños empresarios se

1 Los autobuses urbanos se llaman "micros", apócope de "microbús", en Santiago de Chile. Entre 1991 y 2005 estuvieron pintadas de amarillo. 
adaptó fácilmente al crecimiento de la ciudad y a la demanda que existía. Los recorridos de los microbuses se fueron poco a poco alargando fruto de la demanda y por lo tanto, el número de buses en las calles aumentó considerablemente. Los microbuses amarillos llegaron a cubrir casi la totalidad de la ciudad, con una frecuencia bastante elevada, que permitía a sus pasajeros recorrer la ciudad en un solo trayecto.

$\mathrm{Al}$ mismo tiempo que los microbuses eran fuente de congestión, ruido y contaminación, éstos comenzaron a librar una batalla diaria por los pasajeros, lo que produjo desorden y caos en la ciudad (Trumper, 2005). Así, el sistema llega a ser duramente criticado tanto por sus usuarios como por los poderes públicos (Díaz et al., 2006).

De este modo se puso en marcha en el año 2007 un nuevo sistema de transporte público $^{2}$ en Santiago, el llamado Transantiago. Este sistema incluía en un principio la extensión de las líneas de metro, la reconfiguración de la red de bus, la integración tarifaria a partir de una tarjeta de pago, la renovación total de la flota de bus, la modernización del sistema de gestión de los operadores y de sus empleados y, al mismo tiempo, la construcción de grandes infraestructuras: vías segregadas, paraderos de buses, estaciones intermodales, etc.

Transantiago no sólo fue presentado como la solución frente a los problemas de los microbuses sino que también era uno de los medios para convertir a Santiago en una ciudad de clase mundial, moderna y competitiva a nivel internacional (Maillet, 2008). De este modo el discurso que portaba este cambio estigmatizaba el carácter caótico de los microbuses amarillos, indignos de una nación que aspiraba a ser parte de los países en desarrollo.

Sin embargo, el eventual caos no se reduce a la falta de organización del sistema de transporte sino que también aparece en sus usos imprevisibles, incluso frente a un sistema muy formal. Es importante distinguir la red, más o menos regulada, de servicios de transporte basada sobre una infraestructura fija, y el uso individual de estos servicios, uso que hemos llamamos prácticas de movilidad cotidiana. Éstas corresponden a las maniobras continuas de los desplazamientos cotidianos de los individuos, lo que remite concretamente a la organización diaria de sus actividades, así como a la realización de estas últimas.

De hecho, las prácticas cotidianas de los usuarios siempre modifican el funcionamiento del sistema que les es impuesto, es decir, transforman el sistema. Un ejemplo de lo anterior son las transformaciones que se observan en particular cuando los sistemas de transporte urbano son poco formales, como en el caso de Santiago antes del año 2007 (Jirón, 2007), en Lima (Avellaneda y Lazo, 2009) o en la Paz y Caracas (Baby-Collin, 2005). Similares "tácticas de movilidad" fueron también estudiadas en los casos de sistemas más formales como el de la ciudad de París (Jouffe, 2007) y remiten a la transformación de la movilidad impuesta por los sistemas de transporte. Por otra parte, los notorios procesos de reestructuración de los sistemas

2 Si bien se trata de un sistema de transporte colectivo donde los operadores son privados, hablaremos de transporte público en referencia a la "revolución" que constituye esta recuperación del sistema de transporte por parte del Estado (Maillet, 2007). Además la cuestión del "servicio público" fue una de las referencias en el discurso de la reforma (Díaz, 2006). 
de transporte de ciertas ciudades latinoamericanas imponen la adaptación de la movilidad de sus habitantes, como en el caso del TransMilenio en Bogotá (Montezuma, 2003), en Ciudad de México (Delgado, 1998) o en Curitiba (Rojas y Mello, 2005). De este modo, resultaría interesante poder replicar el presente estudio a estas ciudades, con el objeto de determinar en qué medida sus habitantes no sólo se conforman a los nuevos sistemas formales sino, al mismo tiempo, los transforman al inventar prácticas de movilidad imprevisibles y/o reprimidas.

Planteamos comprender las prácticas de movilidad como maneras de conquistar y apropiarse del territorio, y en particular maneras de adaptar el sistema de transporte o adaptarse a él. En este sentido, se trata de relaciones de poder entre el sistema de transporte y sus usuarios, en la medida que el sistema tiende a imponer un tipo de movilidad al usuario y que éste tiende a transformar el sistema a través de sus prácticas. No pretendemos juzgar la intencionalidad de los usuarios ni buscar un sujeto individual o institucional que ejerce el poder a través del amplio sistema de transporte. Seguiremos la propuesta de Michel Foucault (2001b, p. 302) para quien "el poder no existe, [...] es en realidad relaciones, un haz más o menos organizado, más o menos piramidal, más o menos coordinado, de relaciones” ${ }^{3}$. En este sentido importa incluir en el análisis la resistencia como una dimensión constitutiva del poder (Foucault, 2001c:421), al mismo tiempo que la capacidad de ésta para transformar el orden urbano (Salcedo, 2002).

En definitiva, lo que se busca poner de manifiesto en este artículo son las prácticas de movilidad cotidianas como lugar de expresión de las relaciones de poder entre los usuarios y los sistemas de transporte. Esto desde el punto de vista de los sujetos más que del discurso dominante que afirma el fin del caos en el transporte público por la puesta en marcha de un sistema más formal.

\section{Fundamentos conceptuales: el dispositivo de movilidad y las prácticas de libertad}

Con el fin de analizar las prácticas de movilidad cotidiana en su manera de utilizar, subvertir o de resistir al sistema de transporte que constituye su soporte material, deseamos ampliar la mirada sobre el sistema. Esto significa determinar la escala de pertinencia de su observación, lo cual nos lleva a considerar no solamente la dimensión material del sistema sino que, al mismo tiempo, su dimensión discursiva.

\section{El dispositivo como ensamblaje de materialidades y discursos}

Los elementos a través de los cuales interactúan los usuarios del transporte público son los componentes más visibles con los cuales sus cuerpos son confrontados cotidianamente: los conductores de bus y los otros agentes, los vehículos, las infraestructuras de circulación, de espera, de control, de pago, de información. El usuario está igualmente en contacto permanente con sus iguales, donde la pertenencia al sistema de transporte

3 Desde aquí en adelante las traducciones de los "dichos" de Foucault son de los autores. Nos apoyamos sobre sus conversaciones porque, así como lo explica Deleuze (1989:155), son "diagnósticos" que nos ayudan a entender la actualidad de los conceptos que usó para hacer su "arqueología del saber": "así trazaba esas líneas de actualización que exigían un modo de expresión diferente del modo de expresión propio de los grandes libros”. 
merece ser cuestionada. Más allá de la sola materialidad, nos interesan los discursos que tienen efectos sobre las prácticas, dimensión menos evidente, sobre todo los discursos implícitos en el funcionamiento de las instituciones que las constriñen.

Parece pertinente el concepto de dispositivo definido por Michel Foucault (2001b:299), quien lo define como "un conjunto completamente heterogéneo, compuesto de discursos, de instituciones, de disposiciones arquitecturales, de decisiones reglamentarias, de leyes, de medidas administrativas, de enunciados científicos, de proposiciones filosóficas, morales, filantrópicas, en resumen: lo dicho y asimismo lo no dicho". Esta definición reivindicando la heterogeneidad, identifica los posibles puntos de reencuentro con el dominio de la movilidad cotidiana urbana.

Una segunda característica del dispositivo es "la naturaleza del vínculo entre estos elementos heterogéneos" (ibíd.). No es tanto un conjunto de aparatos sino su ensamblaje, como lo destaca Pløger (2008). Este autor identifica tres problemáticas, que han surgido al aplicar este concepto en el dominio del urbanismo. En particular nos interesa destacar que en la planificación urbana se hace visible una relación entre los discursos y la materialidad. Aparece la importancia de la dimensión discursiva que articula los diferentes elementos, en la interfase del discurso producido y su interpretación por el sujeto en las prácticas cotidianas.

Foucault subraya que "para $[\mathrm{su}]$ cuestión del dispositivo, no es muy preciso decir: acá está lo discursivo, ahí está lo no discursivo” (ibíd.:301). Así no proponemos ni buscamos una estructura discriminando sus elementos heterogéneos.

Diremos por ejemplo que las prácticas cotidianas de los usuarios seguirán la localización de la parada del bus tanto como los "buenos modales" serán reafirmados en la mirada de los otros usuarios. En este sentido, el concepto de dispositivo nos propone cuestionar la combinación de todas estas influencias en vez de aislarlas. Con tal fin, identificamos los dispositivos que regulan la movilidad cotidiana a través de los discursos que articulan entre ellos los elementos materiales e inmateriales del sistema de transporte.

\section{Relevancia del dispositivo en el dominio de la movilidad}

El concepto de dispositivo no sólo se puede aplicar a la movilidad cotidiana, sino que tal aplicación nos parece relevante. Siguiendo la postura foucaldiana, tratamos de "ver el juego y el desarrollo de realidades diversas que se articulan entre sí: un programa, el vínculo que lo explica, la ley que le da coacción, etc., como realidades (aunque bajo otro modo) al igual que las instituciones que le dan cuerpo o como los comportamientos que se añaden con más o menos exactitud" (Foucault, 2001e:847). Nos interesamos en la dinámica propia a este ensamblaje de elementos, que constituye la tercera característica del dispositivo. Después de una génesis orientada por una cierta función estratégica, la evolución del dispositivo produce nuevos objetivos estratégicos para su funcionamiento (Foucault, 2001b:299300). La adaptación de este concepto para entender la evolución de las relaciones de poder otorga una relevancia prospectiva a nuestra problemática. Pensamos que una genealogía del dispositivo de movilidad permitiría acotar con más pertinencia 
dos conceptos supuestamente bien identificados, que son el transporte, en sus infraestructuras técnicas, y la movilidad, en la vida cotidiana, aclarando la dinámica de ésta y su posible evolución.

Desde esta perspectiva la relevancia del dispositivo de la movilidad se traduce en la siguiente hipótesis: el transporte parece ser el nodo del discurso y del funcionamiento del dispositivo de movilidad, y a la vez la idea sobre la cual el dispositivo de movilidad "se conectó hasta cubrir y casi ocultarlo" como "formación a la vez discursiva e institucional" (ibíd.), pero en realidad la idea del transporte fue un producto de este dispositivo que se constituyó primero como discurso sobre otros elementos heterogéneos.

La relevancia de una aplicación del dispositivo al dominio de la movilidad aparece cuando se confronta a las características de dispositivos genéricos o específicos de otros dominios. Por ejemplo, los dispositivos, en particular el de la salud, tienden a generar con la seguridad una doble dependencia, por integración o por marginalización (Foucault, 2001f:1187-1188). Los grandes sistemas de transporte generan efectos similares. El monopolio radical (Illich, 1973) de los medios motorizados de transporte tiende a excluir de aspectos esenciales de la vida cotidiana, a las personas que no quieren o no pueden usar estos medios. A esta marginalización de los sin-auto, la dependencia del automóvil (Dupuy, 1999) añade la dependencia de los con-auto, que organizan su vida cotidiana a partir de la eficiencia que este sistema provee. En este ejemplo, la comparación entre dispositivos de salud y movilidad sugiere analizar problemas de exclusión en términos no sectoriales y buscar en la salud mecanismos relevantes para el análisis del caso de la movilidad.

No consideramos, como varios autores lo hacen (Beuscart, 2006), el dispositivo como una configuración de recursos heterogéneos, lejos de su uso inicial para describir la gubernamentalidad, es decir, el control de la población como una masa representada por cifras (Foucault, 2001a:18). En efecto, uno de los parámetros de ésta es la repartición espacial (ibíd.) que se ejerce en particular a la escala urbana en la medida que "la ciudad con sus principales variables aparece como un objeto que debe ser medicalizado" (ibíd.:22). El vínculo entre los dispositivos de gubernamentalidad y la movilidad se hace a través de dispositivos policiales de salud y vigilancia que producen la ciudad higienista y panóptica (Pløger, 2008). Así la movilidad constituye un dominio de aplicación de dichas técnicas que movilizan más dispositivos que la disciplina moral antigua: "Esas imposiciones de principios morales no son necesarias hoy en día: el prestigio del automóvil, la política de los equipamientos o el incentivo al consumo permiten conseguir normalización de comportamientos muy eficaces" (Foucault, 2001h:1590). La valorización del auto, la proximidad y la accesibilidad a los equipamientos, a los centros de consumo masivo en simbiosis con las carreteras urbanas, apuntan a la movilidad cotidiana como un dominio estratégico para los dispositivos que controlan la población. 
La mirada de lo cotidiano a través del concepto de dispositivo sustituye el léxico de las dificultades concretas del individuo por el del gobierno y la dominación. Permite entonces una mirada sistémica sobre las prácticas de movilidad cotidiana, es decir, usar las prácticas individuales para analizar el sistema de control de la población. La extensión y segregación de las metrópolis tanto como la flexibilidad laboral, están estrechamente asociadas con el desarrollo de sistemas de transporte que necesitan dispositivos para funcionar eficientemente. La movilidad se vuelve entonces el instrumento decisivo de la fragmentación de los territorios y tiempos de la vida cotidiana (Le Breton, 2004), que disuelve al mismo tiempo los lugares y tiempos de la proximidad colectiva potencialmente reivindicativa. Además, se impone como un capital (Kaufmann, 2004) que cada sujeto debe desarrollar y explotar en la competencia socioeconómica individualista actual. Finalmente surge otra idea por vincular a la hipótesis de un dispositivo de movilidad: la movilidad no es destructora de las identidades ligadas al arraigo, sino más bien un proceso más de individualización (Foucault, 2001d). La movilidad como generadora de sus propias formas identitarias (Jouffe, 2010) podría entonces tener más peso en el control de la población como nuevo dispositivo de individualización.

Hablar de dispositivos de movilidad cotidiana supone entonces la existencia, en el ámbito de la movilidad urbana, de dispositivos restrictivos, en el sentido de dispositivos de control, a través de los cuales el poder se ejerce y se resiste, es decir, a través de los cuales las relaciones de poder se concretizan. Por consiguiente, hablar de dispositivos de movilidad es cuestionar la naturaleza de estas relaciones de poder que toman la forma de prácticas cotidianas.

\section{Varios dispositivos de movilidad}

Siguiendo lo anterior nos parece adecuado calificar los discursos de la puesta en marcha del actual sistema de transporte urbano en Santiago como elementos articuladores de un dispositivo de movilidad, especificando varios dispositivos. Esta aproximación está fundamentada por el hecho que este conjunto de discursos es multiescalar, restrictivo y articulador de elementos materiales e inmateriales.

Primero, a través del discurso que dio nacimiento al Transantiago, Maillet (2008) descifra el sistema subyacente de creencias que muestra como hegemónicas. Aunque no toman directamente en cuenta la calidad de vida del usuario, estas creencias pueden ser traducidas por principios de conducta implícitos pero restrictivos para el usuario. Pueden entablar un dispositivo en el sentido ya destacado de dispositivo de control.

Segundo, los discursos de la llegada del Transantiago son más o menos englobantes, portadores de valores generales pero también de orientaciones de políticas públicas y de elecciones técnicas. Por su multiplicidad de escalas, constituyen un marco que articula las varias escalas de las prácticas cotidianas individuales.

En efecto, las prácticas individuales se ejercen también a varias escalas, desde los proyectos vitales vinculados a la materialidad de las oportunidades accesibles en la 
ciudad hasta las exigencias inmediatas frente a la materialidad de las máquinas del sistema del transporte y sus reglamentos concretos (Jouffe, 2007). Aparece entonces, y en tercer lugar, que los discursos en cuestión articulan a cada escala elementos materiales e inmateriales.

A continuación, intentaremos explicitar las creencias subyacentes de los discursos fundadores del Transantiago a partir del análisis multiescalar de Maillet (2008), con el fin de calificar a cada escala relevante lo que llamaremos un dispositivo de la movilidad cotidiana.

Cabe destacar que no hablamos de un dispositivo de movilidad sino de varios. Su distinción se justificará porque cada dispositivo lleva su propia lógica, potencialmente antagonista a otro. Sin embargo, todos apuntan a influenciar las prácticas de movilidad a varias escalas y en distintos dominios de la vida cotidiana. Dado que las escalas y dominios de la movilidad cotidiana están integrados en las decisiones individuales, como en el caso de los trabajadores temporales de la región parisina quienes deben negociar como un todo su "trabajo-desplazamiento" (Jouffe, 2007), los dispositivos que influencian estas decisiones también se encuentran integrados, desde el punto de vista del individuo y de los actores que lo toman en cuenta. En este sentido, se puede hablar de un dispositivo de movilidad en términos genéricos y del dispositivo de movilidad como el conjunto de los dispositivos de movilidad específicos.

\section{Los tres dispositivos de la movilidad cotidiana en Santiago de Chile}

Las tres escalas analizadas por Maillet (2008), es decir, los valores, las políticas públicas y las elecciones técnicas nos permiten proponer tres dispositivos de movilidad para objeto de nuestro análisis.

La valorización de la imagen de la capital y de su transporte de clase mundial asociados al desarrollo y a la modernización del país, sugiere que el usuario debería también defender estos objetivos. En la medida que la modernización está asociada a la puesta en marcha del libre mercado (Maillet, 2008), aparece entonces el dispositivo de trabajo-desplazamiento en su modalidad neoliberal en el sentido de Desjardins (2008). Este autor identifica en las políticas de vivienda europeas la difusión de esta misma modalidad de gobierno. La califica a partir de Foucault como la lucha contra la exclusión para la participación controlada en la economía, opuesta a la modalidad liberal que compensa las desigualdades creadas por el mercado. Caracterizaremos entonces el dispositivo de trabajo-desplazamiento por esta exigencia de participar en la economía para el desarrollo del país.

Estas creencias encuentran su aplicación en las políticas públicas de la integración del sistema de transporte y de la profesionalización del sector. El usuario será entonces invitado a dejar los modos de transporte no integrados y a comportarse como consumidor para que sus elecciones racionales mejoren los estándares de los operadores. El estudio de López et al. (2009) revela también este estatus de consumidor pasivo y no participativo que les da la reforma. El dispositivo correspondiente parece entonces englobar al transporte urbano, público y privado. Sin embargo, López et al. (2009) identifican una diferenciación entre usuarios. El discurso y las 
inversiones públicas sugieren, en efecto, que el usuario del transporte público es considerado como un ciudadano pobre de segunda clase. Esto es coherente con su marginalización en los discursos dominantes (Maillet, 2008). Así, el dispositivo de transporte urbano induce al uso pasivo de los sistemas formales de transporte pero según una modalidad socialmente segregada, el transporte público, siendo el modo de transporte para los sujetos marginalizados del proceso de modernización.

Finalmente, se trata de la puesta en práctica de políticas modernizadoras vía el sueño del autofinanciamiento del transporte público por medio de los propios usuarios, de la baja inversión del Estado y del mejoramiento de la calidad de vida de los ciudadanos por la eficiencia del sistema. El usuario es reducido a una fuente de ingreso, como ya lo era con los microbuses pintados de amarillo (Maillet, 2007:134), que no se beneficiaba de la ayuda estatal pero sí de su propia participación para la eficiencia del sistema. Este "usuario abstracto" (López et al., 2009), agente económico sin corporalidad, aceptará los múltiples inconvenientes concretos de la reforma contra una ganancia en el tiempo de desplazamiento. En esta escala, el dispositivo de transporte público ordena al usuario a seguir las reglas de los operadores, y sobre todo a pagar, atribuyéndole la responsabilidad del mejoramiento de su calidad de vida.

De esta manera los tres dispositivos de la movilidad cotidiana identificados: dispositivos de trabajo-desplazamiento, de transporte urbano y de transporte público, constituyen las aristas a partir de las cuales vamos a intentar analizar las relaciones de poder expresadas en las prácticas cotidianas.

\section{Tres tipos de prácticas como relaciones de poder}

Con fin de describir y analizar las prácticas de movilidad cotidiana como relaciones de poder entre usuarios y dispositivos de la movilidad, nos parece adecuado calificarlas en función de su modalidad de resistencia a los dispositivos. La reflexión de Foucault sobre la gubernamentalidad actual nos ofrece los conceptos adecuados.

En su análisis del "cuidado de sí" que sitúa en el corazón de la ética antigua, identifica esta última con la práctica reflexiva de la libertad (Foucault, 2001g:1530). Ésta y la resistencia que ella permite, son las condiciones de existencia de las relaciones de poder definidas como estrategias para orientar la conducta del otro (ibíd.:1546). El análisis de la práctica de la libertad constituye entonces una manera de observar las relaciones de poder desde las prácticas.

La preocupación por sí mismo se transforma en el conocimiento de sí mismo y en la incorporación de principios y de reglas de conducta según "esquemas que éste encuentra en su cultura y que le son propuestos, sugeridos, impuestos” (ibíd.:1538). Sin embargo, su estudio de la ética de los placeres afirma la autonomía entre esos principios y reglas, y la ética vista como una manera de comportarse bien, en función de las circunstancias (Potte-Bonneville, 2004). Observamos de este modo la manera que tienen los sujetos de resistir o de integrar los principios y las reglas de conducta sostenidas por los dispositivos de movilidad cotidiana, lo que constituiría sus prácticas de libertad. 
Foucault distingue esta categoría a la de liberación, aunque pareciese que a veces puedan confundirse. Estas últimas representan una "condición política o histórica para la práctica de libertad” (Foucault, 2001g:1530), condición a veces necesaria pero nunca suficiente. Constituyen un cuestionamiento al estado de dominación, es decir, a las relaciones de poder asimétricas que vienen a limitar o evitar las prácticas de la libertad. De este modo es que las asimilamos con aquellas prácticas que tienden a la alteración de los dispositivos de la movilidad cotidiana, en la medida que los confundimos con las "técnicas gubernamentales" susceptibles de crear o de mantener tal dominación (ibíd.:1547).

Este autor señala que las prácticas de libertad pueden estar extremadamente restringidas en un estado de dominación. Proponemos entonces identificar un tipo particular de prácticas de libertad que pueden ser reveladoras de tal estado. Nos interesan porque parecen afirmar la imposibilidad de cuestionar la dominación, al revés de las prácticas de liberación. Con el fin de evitar la dura tarea de determinar la efectividad de un estado de dominación, nos interesaremos simplemente en las prácticas reales que corresponden a aquellas que los dispositivos de la movilidad cotidiana pueden inducir directamente. Esta simplificación nos hace asimilar los dispositivos como potenciales generadores de la dominación.

En la medida que estas prácticas constituyen un movimiento que respeta las leyes del dispositivo que las orientan sin afectarlas, a la manera de la libración, es decir, del balanceo regular de un péndulo o de la luna que sigue las imprescindibles leyes de la gravitación universal, las llamaremos prácticas de libración.

Obtenemos finalmente tres categorías de prácticas caracterizadas por su relación con los dispositivos de la movilidad cotidiana: el cuestionamiento liberatorio, el esparcimiento libertario y el respeto estricto; las llamamos respectivamente prácticas de liberación, de libertad y de libración. Finalmente, no deseamos criticar en forma abstracta la adecuación de las categorías definidas más arriba con la realidad diversa de las prácticas de movilidad posibles, sino más bien nos limitaremos a su discusión empírica.

\section{Diversidad de prácticas de movilidad en El Castillo}

El siguiente apartado tiene como objetivo dar cuenta de las prácticas de movilidad de los individuos en su relación con el sistema de transporte. Nos interesa poner de manifiesto el margen de maniobra que tienen los diferentes individuos en relación con lo que el sistema actual les está imponiendo como dispositivo múltiple.

El análisis se apoya en algunos de los resultados del terreno realizado en el mes de julio 2008 en la comuna de La Pintana, a 18 kilómetros del centro de la ciudad, en la parte sur de la ciudad de Santiago de Chile. En esta oportunidad se realizaron 20 entrevistas en profundidad a los habitantes del sector El Castillo. Esta comuna, y este sector en particular, fueron parte de una fuerte política de erradicación de asentamientos informales hacia la periferia, iniciados bajo la dictadura militar entre los años 1970-1980. 
Debemos decir que esta doble marginación, social y espacial, fue reforzada luego de la puesta en marcha del Transantiago, ya que la oferta de transporte de esa zona se degradó, lo que obligó a los pobladores a adaptar sus prácticas de movilidad (Avellaneda y Lazo, 2009). En efecto, la población de La Pintana depende del transporte público para la realización de gran parte de sus actividades cotidianas, ya que no disponen de una oferta de empleos y de servicios de proximidad y la tasa de motorización se encuentra entre las más bajas de la ciudad ${ }^{4}$. Es así que algunas de las prácticas de estos pobladores confrontados a las dificultades de desplazamiento nos permiten comprobar la relevancia del aparato conceptual antes expuesto.

\section{Liberación: del microbús al auto}

Óscar e Isabel son una joven pareja con tres hijos. Viven en el sector del Castillo hace más de veinte años. Esta familia decidió hace muy poco comprar un automóvil, para lo cual pidieron un préstamo bancario. La decisión no fue fácil ya que Óscar es el único que trabaja de la familia y su salario es bastante bajo. A pesar de esto, el deseo de mejorar sus desplazamientos fue más fuerte, tomando la decisión de endeudarse.

Este caso corresponde a lo que consideramos una práctica de liberación frente al dispositivo del transporte urbano. La relación de esta familia con el dispositivo no va a cambiar enteramente a partir de la compra del auto, porque continuarán tomando y esperando el microbús y usando el automóvil sólo para acercarse al metro o a los buses más eficientes llamados "troncales".

A pesar de seguir usando el sistema de transporte público, se liberaron de la dependencia que el dispositivo impone a los pobladores desfavorecidos como ellos. El simple hecho de tener un auto constituye entonces una práctica de liberación. El cambio de estatus que tienen frente a sus vecinos no sólo revela que esta compra genera un margen de libertad mayor al que tenían anteriormente. Se revelan sobre todo sus atributos modales de pobladores desfavorecidos, a través de una norma vivida de lo ordinario que en este caso es modificada a través de la compra del automóvil.

Esta familia que sufre el monopolio radical (Illich, 1973) de los modos de transporte motorizados y en particular del automóvil que estructura la metrópoli santiaguina, percibe el acceso al auto como una liberación que va a aliviar sus dificultades de desplazamiento cotidiano. Pero, siguiendo a los autores de la dependencia automóvil (Dupuy, 1999), la compra del auto puede ser considerada como el comienzo de una dependencia al automóvil mayor que la que tenían con el sistema de buses, en la medida que ciertos desplazamientos realizados en auto no podrán ser hechos en transporte público. Entonces no se trataría tanto de una liberación, más bien podríamos interpretarlo como una alienación al dispositivo del transporte automóvil, que el dispositivo de transporte urbano reserva a las clases acomodadas.

4 Datos de la encuesta origen destino del año 2001. Esta encuesta se realizó entre el período julio 2001 y abril 2002, con el fin de conocer los horarios, los motivos y destinos de los viajes y los medios de transporte elegidos de los habitantes de la capital. La muestra aleatoria incluye 15.000 hogares, que equivalen aproximadamente a 60.000 personas, más los ocupantes de 150 mil vehículos encuestados en lugares estratégicos de la vía pública, La encuesta cubre las 34 comunas del Gran Santiago y zonas como Pirque, Calera de Tango, Lampa y Colina. 
Sin embargo, el uso del auto por esta familia no respeta tampoco el uso individual y exclusivo dado por este dispositivo. Por una parte, el uso es altamente intermodal. El auto sirve de conexión con las líneas de micros más frecuentes, permitiendo completar el sistema de buses, y no de sustituirlo. Por otra parte su uso es colectivo, tal como lo observa Le Breton (2008) en el caso de familias de escasos recursos en Francia. El jefe de familia, que sabe conducir, lleva a los otros miembros del hogar que lo necesitan. Pero, además de su uso familiar, el auto es puesto a la disposición de los vecinos. Así es que no se trata sólo de una práctica de libertad que toma distancia del dispositivo. Esta disponibilidad local del automóvil familiar produce una fuerte transgresión y una liberación del sistema bimodal, oponiendo modo público colectivo y modo privado individual, en la medida que se crea un microsistema de transporte alternativo, colectivo, local y solidario.

\section{Liberación: escaparse del trabajo para escaparse del transporte}

José es maestro de la construcción y está cesante. Él ha trabajado toda su vida en el sector más acomodado de la capital. Antes realizaba largos trayectos en microbús para llegar a su trabajo y eso no le causaba mayor problema, pues sólo debía tomar un microbús. Actualmente, y a causa de los cambios en el transporte, este hombre ha reducido su radio de búsqueda de trabajo, limitando su búsqueda a los lugares donde sea fácil llegar desde su hogar.

La contradicción de este habitante con el sistema de transporte se torna visible cuando decide rehusar las ofertas de empleo porque ellas se encuentran muy lejos de su lugar de residencia actual. La transgresión con aquello que le impone el dispositivo trabajo-desplazamiento, es decir, ir a trabajar a pesar de las dificultades de trabajo o de desplazamiento, es patente: este hombre dice que no le importa tener que pasar un poco más de tiempo cesante y "apretarse el cinturón” si esto significa poder ir a trabajar más cerca. Prefiere asumir lo que significa estar cesante antes que desplazarse durante largas horas, hacer correspondencias y sufrir la incomodidad de los desplazamientos diarios.

Él se excluye de una parte importante del mercado de trabajo. En este sentido hay una transgresión de la exigencia neoliberal de participación a la economía. Su transgresión del dispositivo trabajo-desplazamiento es doble, rehusando a la vez desplazarse y trabajar.

José piensa que si encuentra un trabajo que le satisfaga en términos de transporte y proximidad, no solamente se sentirá mejor, sino que también significará una ganancia de tiempo importante que podrá ocupar en otras tareas que beneficiarán a su familia en el largo plazo. Así, este hombre continuará esperando encontrar un trabajo que le convenga y que esté más o menos cerca de su hogar. Él demuestra su liberación de los principios que rigen la movilidad cotidiana y el trabajo.

\section{Liberación o libertad: cambiar de trabajo para cambiar de transporte}

El caso de Betty es similar y corresponde también a una práctica de liberación en su relación con el dispositivo de trabajo-desplazamiento. Betty llegó al Castillo hace 
19 años y acá nacieron sus 7 hijos. Tiene un puesto en la feria de la población donde vende "ropa americana". Sin embargo esta mujer no eligió ser feriante, más bien se debió a los problemas que tenía para llegar a su trabajo anterior. Antes ella debía desplazarse en micro para ir a trabajar, lo que le costaba dinero y bastante tiempo. Esta situación la impulsó a buscar un trabajo independiente en su propia comuna, pudiendo hacer los desplazamientos a pie.

'Primero trabajaba aquí en el consultorio, es cerquita sí, no tenía problema (...) pero cuando cambió esto yo ya empecé a tener problemas, porque yo me iba a la misma hora y llegaba media hora o una hora más tarde (...) seguí ahí hasta que, un día decidí, me pagaron y les dije: yo no voy a trabajar más les dije yo, porque hay mucho problema con la locomoción".

Betty adaptó su configuración de trabajo-desplazamiento pero no dejó de trabajar. Por lo tanto, podríamos considerar la elección de Betty como una libertad respecto al dispositivo de trabajo-desplazamiento: no rompe con éste como lo hace José, y tampoco se somete estrictamente a su principio, es decir, poner su movilidad al servicio de la economía. Sin embargo, Betty escogió un trabajo muy informal en la feria, un puesto de "cólera", es decir, al final de la feria, en la cola, donde la gente no tiene permiso legal para tener un puesto. De esta forma, esta trabajadora se liberó de la exigencia de participación controlada a la economía. La adaptación del empleo de Betty a sus dificultades de traslado corresponde entonces también a una liberación en relación con el dispositivo de trabajo-desplazamiento.

\section{Libertad: abandonar el microbús alimentador para continuar tomando los buses troncales}

Los modos de transporte no motorizados, principalmente la caminata y la bicicleta, han sido por largo tiempo analizados como recurso para las poblaciones pobres. $\mathrm{Su}$ uso para acceder a la red de transporte público correspondería a lo que nosotros hemos llamado prácticas de libertad en su relación con el dispositivo de transporte urbano. De este modo, para la población observada, los modos de transporte no motorizados se constituirían en un modo de transporte alternativo o secundario cuando el microbús no pasa. Acá se habla de los buses alimentadores, que conectan de manera poco eficaz los barrios con las estaciones de metro o con las grandes líneas estructurales de Transantiago, que pasan al exterior de las zonas residenciales.

Dicho de otra manera, algunos individuos van a movilizar sus capacidades de adaptación para utilizar el sistema de transporte público, como por ejemplo la transformación de la bicicleta o la caminata en modo importante para salir del barrio. Ellos se pondrán de esta forma en relación con el dispositivo de transporte urbano a partir de un respeto distante de las reglas que les impone el uso del transporte público. No aplican la regla de la utilización de los buses alimentadores, lo que sería una libración, pero tampoco salen totalmente del sistema de transporte público, lo que sería una liberación. Se trata de una práctica de libertad. 
Mario es un adolescente que vive en el Castillo. Para este joven el problema de transporte se traduce en poder salir y entrar a la población. El problema para él no es la falta de transporte y de buses troncales, sino que más bien son los microbuses que alimentan el barrio los que son un problema. "Una vez afuera, es fácil desplazarse en micro o en metro", dice. La tarde antes de la entrevista, después que Mario regresaba de su escuela, decidió pasar a un concierto que se realizaba en la misma comuna, pero fuera de su barrio. El concierto terminó tarde en la noche y Mario se encontró sin saber cómo volver a su casa. Él estaba al mismo tiempo cerca pero lejos de su hogar. Como muchos otros jóvenes de la población, Mario dejó de hacerse preguntas sobre cómo regresar a su casa. Prefiere hacerse la idea de que es difícil que pase el microbús por lo que camina hasta su hogar.

El caso de Juan es bastante similar. Para él la caminata es una práctica importante dentro de sus desplazamientos cotidianos. Juan comenzó levantándose entre las 5:00 y las 5:30 de la mañana para poder "atrapar" el microbús que pasaba cerca de su casa. Finalmente se cansó de esperar, ya que se dio cuenta que no servía de nada levantarse más temprano, ya que de todas formas terminaba caminando una media hora para tomar el microbús troncal que pasaba fuera de la población.

Si bien caminar desde su casa hasta la parada de los buses troncales es un camino largo y agotador, sobre todo de regreso en la tarde, Juan prefiere hacer esto y no tener que estar esperando largo tiempo a que el microbús pase.

Las prácticas de Mario y Juan constituirían entonces prácticas de libertad porque permiten salir de lo que impone estrictamente el dispositivo de transporte urbano al someterse a su principio general, utilizar el transporte público. Comprenderemos entonces el concepto de libertad como una salida (exit en el sentido de Hirschmann) parcial del sistema, eventualmente una salida del subsistema de microbuses alimentadores, gracias a la puesta en marcha de prácticas de desplazamiento cortas y complementarias.

\section{Libración: anticipar y optimizar}

Observamos otro grupo de prácticas que manifiestan un grado de disidencia menor frente al dispositivo de transporte público. Dicho de otro modo, se trataría de personas que se limitan a lo que es impuesto por el dispositivo de transporte, reduciendo así su margen de maniobra. Estos individuos se ven en la obligación de optimizar sus modos de desplazamientos, así como sus momentos y lugares de actividad. De esta forma este grupo de personas entraría en lo que llamamos prácticas de libración.

El caso de María ilustra bien esto. Se trata de una mujer que trabaja como asesora de hogar en la comuna de La Florida. Su día comienza a las 9:30 de la mañana hasta las 16:30. Para ella su lugar de trabajo no está lejos y tampoco le parece que sea difícil llegar a él. María toma el microbús hasta el metro, en un trayecto que puede durar una hora. Cuando tiene algún problema no duda en tomar un taxi colectivo. Este caso, correspondería a otra variante de adaptación a la insuficiencia de los buses alimentadores, es decir, de una práctica de libertad frente al dispositivo de transporte urbano en la medida que éste es concebido como autosuficiente y por lo tanto independiente de los otros modos. 
Si bien ella no tiene problema para llegar a su trabajo, a veces le da un poco de "flojera" tener que salir para hacer otras actividades. María siente que no sabe llegar a los lugares que frecuentaba regularmente antes del Transantiago. En consecuencia, ella planifica con bastante antelación sus salidas hacia otras comunas, sobre todo cuando se trata de visitar a su familia, preguntando, verificando el trayecto, para poder llegar sin dificultad al destino deseado.

Para objeto de nuestro análisis diremos que María pone en marcha la práctica de libración en el sentido que trata de organizar y de optimizar sus trayectos y salidas sin que las restricciones que ella asume por entero se traduzcan en algún tipo de contradicción.

\section{Libración: trabajar más para lograr desplazarse}

Otra práctica de libración pero diferente a la anterior es la de Rosa, quien hace la limpieza en un centro comercial. Esta mujer hace doble turno de trabajo. En un principio trabajaba hasta medianoche, pero luego decidió continuar con otro turno hasta las 7 de la mañana, ya que le era bastante difícil regresar a medianoche a su hogar.

En este caso se trataría de una práctica de libertad frente al dispositivo de transporte público, una adaptación innovadora pero respetuosa del sistema de transporte público gracias a una espera trabajosa. Se trata más bien de una libración en relación con el dispositivo de trabajo-desplazamiento porque ella se encuentra "atrapada" por las condiciones de trabajo y la sola opción que le queda es adaptarse y optimizar de manera forzada sus horas de trabajo, pero en el sentido de un celo en su participación en el mercado de trabajo. De esta manera, la libración de Rosa se opone directamente a la liberación de José, ya que este último rehúsa el principio de inserción económica a todo precio.

\section{Libración: denunciar el fraude}

Finalmente nos parece interesante revelar que las relaciones de poder se manifiestan también en las prácticas discursivas que justifican las prácticas de movilidad. Así, un hombre que critica fuertemente el fraude de los usuarios del transporte entra en lo que hemos llamado una práctica de libración en la medida que defiende, en este caso, la regla de pagar el boleto de bus. Se opone al fraude en tanto que práctica de liberación que transgrede una regla mayor y explícita del dispositivo de transporte público y lo amenaza financieramente.

"Entonces, el Transantiago no es malo; que no nos cae, no nos quede bien a algunas personas, pero hay algunos que los ha favorecido (...) pero no se trata de un mal servicio, si este servicio no funciona es por falta de dinero porque la gente no paga, no paga."

La práctica discursiva de libración de este hombre va hasta el punto de justificar la eficiencia global del principio de Transantiago. Se pone a los que se suben sin pagar como los responsables de que el sistema no funcione y se justifica de este modo la legitimidad de esta regla del dispositivo de transporte público. 


\section{Conclusión: hacia un análisis político de las prácticas de movilidad}

A lo largo de este artículo hemos querido poner en evidencia la hipótesis por medio de la cual las prácticas de movilidad cotidiana concretizan relaciones de poder entre sujetos y dispositivos. Dicho de otra forma, los dispositivos de movilidad constituirían un dispositivo de control de la población en el contexto metropolitano actual.

La construcción de la hipótesis de un dispositivo de movilidad para Santiago de Chile debe también preguntarse por la estructura de este dispositivo múltiple. En este sentido, hemos podido salir de la aproximación sectorial del transporte para subrayar la importancia de la relación del trabajo en la comprensión de la movilidad cotidiana. Aparecieron así en nuestro análisis tres escalas: los dispositivos de movilidad de desplazamiento-trabajo, de transporte urbano y de transporte público. Sin embargo, y para completar nuestro estudio, se propone integrar en un futuro a otros subsistemas de relaciones de poder, integrando los desplazamientos y los diversos dominios de la vida cotidiana como por ejemplo el consumo.

Además, la caracterización de las prácticas de movilidad como prácticas de liberación, libertad y libración, es decir, su calificación en función del grado de resistencia o sumisión a los distintos dispositivos, permite entender la capacidad subversiva de los usuarios, la eficiencia de los dispositivos y las interacciones entre éstos. Finalmente podemos decir que la mirada foucaldiana a la movilidad bajo la forma del análisis de la formación y de la evolución estratégica de sus dispositivos específicos, ofrece amplias perspectivas para descifrar las prácticas urbanas frente al marco restrictivo de su realización cotidiana.

Por otra parte y para el caso específico de nuestro estudio, podemos destacar puntos claves de articulación entre las prácticas y los dispositivos de movilidad. Primero, la bicicleta y la caminata en reemplazo de los buses alimentadores aparecen como prácticas de libertad frente al dispositivo de transporte público, mientras la planificación de los viajes tanto como la denuncia del fraude serían libraciones de éste. Segundo, el uso intermodal, colectivo, solidario y local del auto se deja ver como una herramienta de liberación del dispositivo de transporte urbano, a pesar de la dependencia que éste pueda generar. En tercer y último lugar, la elección del trabajo se afirma como una modalidad mayor de las relaciones de poder en la movilidad cotidiana. Elegir un trabajo más largo y agotador constituye una libración respetuosa del dispositivo de trabajodesplazamiento, mientras que un trabajo más cercano permite una libertad y la cesantía la liberación.

Pensamos que este tipo de pauta de lectura, apuntando a la definición de dispositivos y a la observación de prácticas como relaciones de poder, abre la puerta para una lectura política de las prácticas cotidianas. De hecho, es posible un 
análisis crítico al sistema de transporte y a los sistemas que lo rodean, es decir, más precisamente, la identificación de un estado de dominación asociado a los dispositivos que pretenden sólo emancipar a las poblaciones. Ello puede llegar a la caracterización de las dinámicas en el dominio de la movilidad cotidiana. No obstante, hay que considerar que cada categoría de prácticas autoriza una doble interpretación. Así, las prácticas de libertad, como la caminata y el uso de la bicicleta en lugar del microbús alimentador, muestran una insuficiencia pero también una flexibilidad del sistema actual de transporte urbano. La interpretación política parece difícil a partir de la simple constatación de la práctica, limitación que caracteriza nuestro estudio. Aparece entonces la necesidad de completar nuestro análisis con una interpretación de las prácticas desde el sujeto y por medio de la observación de sus condiciones de existencia. IEURE

\section{Referencias bibliográficas}

Avellaneda, P. \& Lazo, A. (2009). Aproximación social al estudio de la movilidad cotidiana en la periferia pobre de la ciudad. Los casos de Juan Pablo II, en Lima, y de La Pintana, en Santiago de Chile. Comunicación presentada en el XV Congreso Latinoamericano de Transporte Público y Urbano, Buenos Aires, 31 de marzo al 3 de abril.

Baby-Collin, V. (2005). Des marges dans la ville: mobilités, citadinités et métissage de l'urbanité. En Capron, Guenola; Cortès, Geneviève. y Guétat-Bernard, Hélène. (2005). Liens et lieux de mobilité. Ces autres territoires. Paris: Belin.

Beuscart, J-S. \& Peerbaye, A. (2006). Histoires de dispositifs (introduction). Terrainse travaux, 2 (11), 3-15.

Deleuze, G. (1989). Qu'est-ce qu'un dispositif? En Michel Foucault philosophe. Rencontre internationale. (pp.185-195). Paris: Seuil.

Delgado, J. (1998). Ciudad-Región y transporte en el México central. Un largo camino de rupturas y continuidades. Universidad Nacional Autónoma de México. México: Plaza y Valdés.

Desjardins, X. (2008). Le logement social au temps du néolibéralisme. Métropoles, 4. Recuperado el 4 junio 2009, de http://metropoles.revues.org/document3022.html.

Díaz, G; Velasco, A. \& Gómez-Lobo, A.(2006). Micros en Santiago: de enemigo público a servicio público. En Galetovic, A. (ed.). Santiago: Dónde estamos y hacia dónde vamos (425-460). Santiago: CEP.

Dupuy, G. (1999). La dépendance automobile. Symptôme, analyse, diagnostic, traitement. Paris: Anthropos. Foucault, M. (2001a). La politique de la santé au XVIIIe siècle. En Foucault, M., Dits et Ecrits 19541988. T. II, Paris: Gallimard, p. 13-27.

Foucault, M. (2001b). Le jeu de Michel Foucault. En Foucault, M. Dits et Ecrits, 1954-1988. T.II, Paris: Gallimard, p. 298-328.

Foucault, M. (2001c). Pouvoirs et stratégies. En Foucault, M., Dits et Ecrits, 1954-1988. T. II, Paris: Gallimard, p. 418-428.

Foucault, M. (2001d). Sexualité et pouvoir. En Foucault, M., Dits et Ecrits, 1976-1988. T. II, Paris: Gallimard, p. 552-570. 
Foucault, M. (2001e). Table ronde du 20 mai 197. En Foucault, M. Dits et Ecrits, 1954-1988. T II, Paris: Gallimard, p. 839-853.

Foucault, M. (2001f). Un système fini face à une demande infinie. En Foucault, M. Dits et Ecrits, 19541988. T. II, Paris: Gallimard, p. 1186-1202.

Foucault, M. (2001g). L'éthique du souci de soi comme pratique de liberté. En Foucault, M. Dits et Ecrits, 1954-1988, T. II, Paris: Gallimard, p. 1527-1548.

Foucault, M. (2001h). Sur la sellette. En Foucault, M. Dits et Ecrits, 1954-1988. T. I, Paris: Gallimard, p. $1588-1593$.

Hidalgo, R. (2007). ¿Se acabó el suelo en la gran ciudad? Las nuevas periferias metropolitanas de la vivienda social en Santiago de Chile. Revista EURE, 98, 57-75.

Illich, I. (1973). Énergie et équité. Paris: Seuil.

Jirón, P. (2007). Implicancias de género en las experiencias de movilidad cotidiana urbana en Santiago de Chile. Revista Venezolana de Estudios de la Mujer, 29 (12), 173-197.

Jouffe, Y. (2010). Attachement et émancipation. Les formes identitaires comme ressources de la mobilité des précaires flexibles, En Ramadier, Th. y Depeau, S (Eds.). La mobilité quotidienne en classes : les identités sociales au regard des pratiques spatiales. (en prensa). Rennes: PUR.

Jouffe, Y. (2007). Précaires mais mobiles. Tactiques de mobilité des travailleurs précaires flexibles et nouveaux services de mobilité. Thèse de doctorat de sociologie. École Nationale des Ponts et Chaussées. Champs-sur-Marne. Paris.

Kaufmann, V; Bergman, M. \& Joye, D. (2004). Motility: Mobility as Capital. International Journal of Urban and Regional Research, 28 (4), 745-56.

Le Breton, É. (2008). Domicile-travail: Les salariés à bout de souffle, Paris: Les Carnets de l'Info.

Le Breton, É. (2004). Les épreuves de la dispersion. Recherche exploratoire sur les expériences individuelles de la société dispersée rapport de recherche pour le Predit 2002-2006, Groupe opérationnel $n^{\circ} 1$ Mobilités, territoires et développement durable.

López, S. \& Hinojosa, O. (2009). Usuarios del transporte en Santiago: ¿Pasajeros o actores políticos? Comunicación presentada en el XV Congreso Latinoamericano de Transporte Público y Urbano, Buenos Aires, 31 de marzo al 3 de abril.

Maillet, A. (2008). La gestación del Transantiago en el discurso público: hacia un análisis de políticas públicas desde la perspectiva cognitivista. En De Cea, M ; Paola Díaz \& Géraldine K (comp.). Chile ¿De país modelado a país modelo? Una mirada sobre la política, lo social y la economía (pp. 325345) Santiago: LOM. Recuperado de http://nuevomundo.revues.org/index10932.html

Maillet A. (2007). Revolución en un campo: el caso de la reforma del transporte público en Santiago. Revista Central de Sociología (2), 133-143.

Montezuma, Ricardo. (2003)Transformación urbana y movilidad: bases para el estudio en América Latina. Quito: Programa de Gestión Urbana. Recuperado de: http://www.ciudadhumana. org/Publicaciones/internacionales/PDF/Transformacion\%20urbana\%20y\%20movilidad. pdfPløger, J. (2008). Foucault's Dispositif and the City, Planning Theory, 7(1), 51-70.

Potte-Bonneville, M. (2004). Politique des usages. Une boîte à outils pour la lutte des usagers, Vacarme, 29. Rojas, F \& Mello, C. (2005) El transporte público colectivo en Curitiba y Bogotá. Revista de ingeniería. (21). Facultad de ingeniería Universidad de los Andes. Bogotá. https://www.bmi.gob.sv/ pls/portal/docs/page/bmi_htmls/bmi_htmls_pulso_transporte/el\%20transp\%20colectivo\%20en\%20curitiba\%20y\%20bogotá.pdf 
Salcedo, Rodrigo (2002). El espacio público en el debate actual: Una reflexión crítica sobre el urbanismo postmoderno. Revista EURE 28 (84).

Trumper, R. (2005). Automóviles y microbuses: construyendo neoliberalismo en Santiago de Chile. En Hidalgo, R.; Trumper, R. \& Borsdorf, A. Transformaciones urbanas y procesos territoriales. Lecturas del nuevo dibujo de la ciudad latinoamericana (pp. 71-81). Pontificia Universidad Católica de Chile. 